\title{
Optimization of Multi-Band DFT-Spread DMT System for Polymer Optical Fiber Communications
}

\author{
Linning Peng, Maryline Hélard, Sylvain Haese \\ Institut d'Electronique et des Télécommunications de Rennes (IETR), UMR 6164 \\ INSA, Université Européenne de Bretagne, 35708 Rennes, France \\ \{linning.peng, maryline.helard, sylvain.haese\}@insa-rennes.fr
}

\begin{abstract}
Recently, polymer optical fiber (POF) became a popular solution for the indoor communications. In this paper, a multi-band discrete-Fourier-transform spread (MB-DFT-S) discrete multi-tone (DMT) system is proposed to study and optimize in terms of POF communications. A joint optimization of used subcarrier number, used bandwidth and multi-band number is investigated. The transmission of MB-DFT-S DMT over $50 \mathrm{~m}$ POF link is implemented. Both theoretical and experimental results demonstrate that optimized MB-DFT-S DMT system outperforms the original DMT and DFT-S DMT systems, which means that it is a promising technique for future POF transmission systems.
\end{abstract}

\section{INTRODUCTION}

High data rate indoor transmission over $1 \mathrm{~mm}$ core diameter poly-methyl-methacrylate (PMMA) polymer optical fibers (POFs) became more and more attractive in recent researches [1]. $1 \mathrm{~mm}$ PMMA POFs own the advantages of high robustness, easy coupling and low maintenance, which is considered to be a cost-effective and do-it-yourself solution for short-range communications and in particular suitable for highspeed communications. Thus POF is studied in the seventh framework programme (FP7) of the European ALPHA Project [2]. An investigation of cost optimization for in-building networks shows that, in a foreseeable future, POF could be the most economic solution for home networking, compared to traditional copper based category 5 (CAT-5E) solution and optical single-mode fiber (SMF) and multi-mode fiber (MMF) solutions [3]. However, due to the numerous modal dispersions, the useful transmission bandwidth of POF is limited compared to other types of optical fiber [4].

A majority of today's short-range optical communication systems are based on ON-OFF keying (OOK). The transmission rate is limited due to POF's $3 \mathrm{~dB}$ bandwidth [3]. Discrete multi-tone (DMT) modulation, which is very similar to the well-known orthogonal frequency-division multiplexing (OFDM), is proved to be a practical solution to approach channel capacity in combination with bit-loading and power allocation techniques in case of frequency selective channel [5]. Compared to OOK modulations, DMT systems require lower ADC sampling rate and allow easier equalization [6]. However, DMT suffers from high peak-to-average power ratio (PAPR) problem. Clipping is shown to be an efficient PAPR reduction approach in recent researches [6][7]. In order to deal with PAPR problem, scheme named DFT-Spread (DFT-S) DMT was proposed and evaluated for POF transmission [8]. DFT-S DMT derives from signal carrier frequency division multiple access (SC-FDMA) that is an attractive technique in wireless communications for 4G mobile standard [9], keeping multi-carrier flexibility and advantage of single carrier systems. It is worth noting that compared to classical DMT systems, DFT-S DMT system requires a robust equalizer in order to mitigate inter symbol interferences. Capacity of SC-FDMA system has been investigated with frequency-domain equalization [10], which can also be suitable for DFT-S DMT schemes. Nevertheless, DFT-S DMT is also sensitive to nonlinearity distortion in optical systems [11], which is a drawback to its practical applications.

Recently, an improved DFT-S OFDM scheme with split DFT-spread blocks has been tested by experiments for SMF with coherent optical fiber system [12]. Results show that multi-band (MB) DFT-S OFDM owns the behavior of strong robustness against non-linearity, which means that it is suitable for practical optical fiber communication system [11].

The aim of this paper is to study and optimize a MB-DFTS DMT scheme for low-cost POF system. It is worth noting that with the help of split bands, bit-loading technique is inherently available for MB-DFT-S DMT schemes. We derive the theoretical POF channel capacities of MB-DFT-S DMT schemes and evaluate the maximum transmission bit-rate. Furthermore, an optimization approach of the number of used subcarrier, used bandwidth and the number of split bands is proposed for MB-DFT-S DMT in POF. Then, the theoretical analysis is verified by the hardware experiment, which is carried out in a MB-DFT-S DMT implementation over a $50 \mathrm{~m}$ POF.

This paper is organized as follows: In section II, the system model is presented including the explanation of the MB-DFT-S DMT principle and the description of the channel model further used. Section III introduces a theoretical bit-rate calculation for POF channel. Section IV expresses PAPR problem of different modulation schemes. Then in Section $\mathrm{V}$, a theoretical optimization of MB-DFT-S transmission over POF channel is developed. Section VI is dedicated to demonstrate "real" experimental results in order to compare with theoretical analysis. Finally, a conclusion is given in Section VII.

\section{SYSTEM MODEL}

\section{A. Principle of $M B-D F T-S D M T$}

We can note that unlike the original MB-DFT-S OFDM with optical I/Q modulator [11][12], MB-DFT-S DMT 


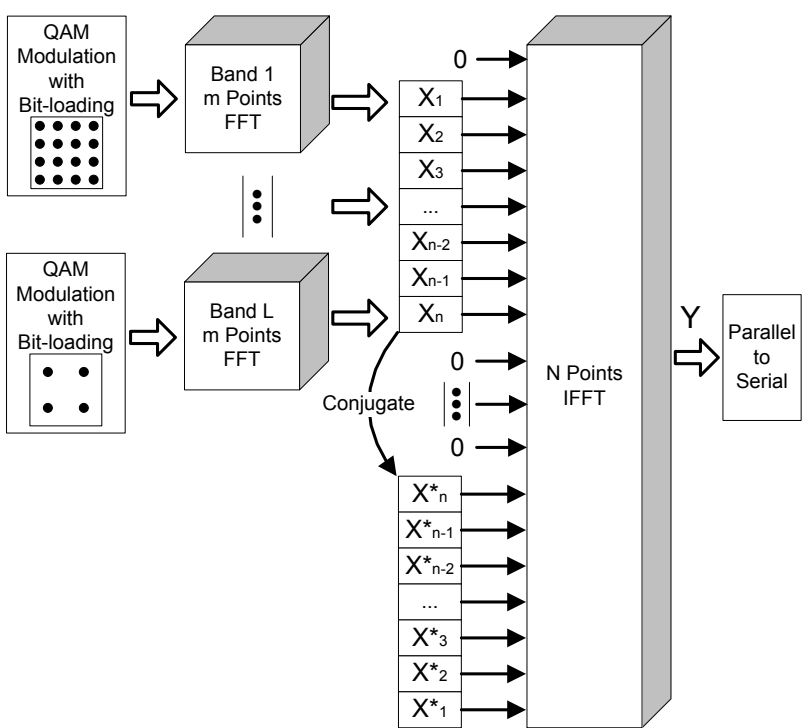

Figure 1. Block diagram of a MB-DFT-S DMT transmission

symbols are directly generated in real values and fed to electrooptic devices, which is suitable for the low-cost POF channel. The block diagram of the baseband MB-DFT-S DMT modulation is shown in Figure 1. As shown in the figure, $L$ bands are allocated in the system and can be assigned with different modulation orders. Then, the different bands are precoded by a $m$ points FFT block respectively. Multi-bands are mapped to different sub-carriers of the whole transmission band. The first subcarrier and subcarriers at high frequency range are allocated with 0 . Finally, conjugated version of the generated symbols $\left\{X_{i}: i=1,2, \ldots n\right\}$ are arranged in an inverse sequence $\left\{X_{i}^{*}: i=n, n-1, \ldots 1\right\}$ and put in the second-half of the $2 N$-points IFFT block. The modulated MBDFT-S DMT symbols can be represented as:

$$
\begin{gathered}
Y=\operatorname{IDFT}\left(0, X_{M}^{1} \cdots X_{M}^{L}, \delta, X_{M}^{L^{*}} \cdots X_{M}^{1^{*}}\right), \\
X_{M}^{l}=\sum_{m=0}^{M-1} x_{m}^{l} \cdot e^{j \frac{2 \pi m n}{M}},
\end{gathered}
$$

where $L$ is the number of considered bands; $M$ is the number of the FFT in each bands; $n$ is the number of used subcarriers, which follows the equation $M \cdot L=n ; \delta$ are null subcarriers in the DMT modulation.

\section{B. POF Channel Model}

The POF channel capacities are defined in the $3 \mathrm{~dB}$ bandwidth of the channel transfer function. One well-known approach of POF modeling is the Gaussian low-pass channel response based on its $3 \mathrm{~dB}$ bandwidth [4]. In terms of Gaussian low-pass channel model in SI-POF, we can calculate channel capacities with channel magnitude response shown as:

$$
\left|H_{i}\right|^{2}=\exp \left[-\left(\frac{f_{i}}{f_{0}}\right)^{2}\right]
$$

where $f_{0}$ is an estimated frequency related to the $3 \mathrm{~dB}$ bandwidth, shown as $f_{0}=f_{3 d B} / \sqrt{\ln 2}$. This POF channel will be used to theoretically calculate the capacities of POF transmission.

\section{CHANNEL CAPACITY OF MB-DFT-S DMT IN POF}

The channel capacity in a communication system is defined as:

$$
C=W \cdot \log _{2}(1+S N R),
$$

where $W$ is the used bandwidth. In the MCM system, in order to investigate the system capacity, we can divide the used bandwidth $W$ into $n$ subcarriers with equal subcarrier spacing. It is worth noting that in case of total output power is limited, the larger the used bandwidth $W$, the less the available power can be allocated in each subcarrier. In the POF transmission system, the maximal optical output power is limited in order to keep the system working in the linear range. Therefore it is significant for evaluating the channel capacity of MB-DFTDMT transmission over POF with different used bandwidth.

In this section, we will firstly remind the channel capacity in DMT and DFT-S DMT systems. Then we derive the channel capacity in our MB-DFT-S DMT system. After that, in order to maximize performance in POF channel, bit-loading algorithms are introduced for MB-DFT-S DMT system.

\section{A. MB-DFT-S DMT Channel capacity}

The capacity of DMT modulation is represented as follows:

$$
C_{D M T}=\sum_{i=1}^{n} C_{i}=\sum_{i=1}^{n} \log _{2}\left(1+\frac{E_{\mathrm{i}} \cdot\left|H_{\mathrm{i}}\right|^{2}}{\sigma_{\mathrm{i}}^{2}}\right),
$$

where $E_{i}$ is allocated power to $i^{\text {th }}$ subcarrier; $\left|H_{i}\right|$ is channel magnitude response of $i^{t h}$ subcarrier; $\sigma_{\mathrm{i}}{ }^{2}$ is noise power at the $i^{t h}$ subcarrier. Under the assumption of equal power allocation for each DMT subcarrier, we can get $E_{\mathrm{i}}=P / n$, where $P$ is total available output power. Furthermore, with the assumption of an additive white Gaussian noise in POF system, $\sigma_{\mathrm{i}}{ }^{2}$ is defined as $\sigma^{2}$.

The channel capacities in DFT-S DMT with zero-forcing equalization are proposed as follows [10]:

$$
C_{D F T-S}=\sum_{i=1}^{n} \log _{2}\left(1+\frac{1}{\frac{1}{n} \cdot \sum_{m=1}^{n} \frac{\sigma^{2}}{\left|H_{m}\right|^{2} \cdot E_{\mathrm{m}}}}\right) \text {, }
$$

In case of MB-DFT-S DMT with multi-band, channel capacities are calculated in each split band and finally summed in the whole band. We merely derive the channel capacities of MB-DFT-S DMT from (6), which can be represented as follows:

$$
\begin{aligned}
& C_{M B-D F T-S}= \\
& \sum_{l=0}^{L-1} \sum_{m=1}^{n / L} \log _{2}\left(1+\frac{1}{\frac{L}{n} \cdot \sum_{m=1}^{n / L} \frac{\sigma^{2}}{\left|H_{m+l \cdot(n / L)}\right|^{2} \cdot \mathrm{E}_{\mathrm{m}}^{l}}}\right),
\end{aligned}
$$

\section{B. Bit-loading in MB-DFT-S DMT}

The available total bit-rate in DMT system employing bitloading is shown as: 


$$
\mathrm{b}_{\mathrm{DMT}}=\sum_{i=1}^{n} b_{i}=\sum_{i=1}^{n} \log _{2}\left(1+\frac{E_{\mathrm{i}} \cdot\left|H_{\mathrm{i}}\right|^{2}}{\Gamma \cdot \sigma_{\mathrm{i}}^{2}}\right)
$$

where parameter $\Gamma$ is the SNR gap to capacity associated with an error rate target. It is related to the modulation map in practical applications. In case of QAM, $\Gamma$ with a target symbol error rate (SER) can be represented as [13]:

$$
\Gamma=\frac{1}{3}\left[\mathrm{Q}^{-1}\left(\frac{\mathrm{SER}}{4}\right)\right]^{2}
$$

In our MB-DFT-S DMT system, the practical bit-rate employing bit-loading is given as:

$$
\begin{aligned}
& \mathrm{b}_{\mathrm{MB}-\mathrm{DFT}-\mathrm{S}}= \\
& \sum_{l=0}^{L-1} \sum_{m=1}^{n / L} \log _{2}\left(1+\frac{1}{\frac{L}{n} \cdot \sum_{m=1}^{n / L} \frac{\sigma^{2}}{\left|H_{m+l \cdot(n / L)}\right|^{2} \cdot \mathrm{E}_{\mathrm{m}}^{1}} \cdot \Gamma}\right),
\end{aligned}
$$

The allocated power in each band can be optimized, in terms of constant sum of all subcarrier powers:

$$
\sum_{\mathrm{l}=0}^{\mathrm{L}-1} \sum_{\mathrm{m}=1}^{\mathrm{n} / \mathrm{L}} \mathrm{E}_{\mathrm{m}}^{\mathrm{l}}=\mathrm{P}
$$

The transmission bit-rate in a practical MB-DFT-S DMT system can be optimized by Chow's [14] algorithms.

\section{PAPR IN MB-DFT-S DMT}

Before investigating the POF channel capacities in MBDFT-S DMT schemes, the PAPR problem is considered in order to calculate the SNR gain for each scheme with fixed maximal optical output power. An investigation of complementary cumulative distribution function (CCDF) of PAPR in DMT, DFT-S DMT and MB-DFT-S DMT schemes are shown in Figure 2. DMT modulation inherits the highest PAPR and DFT-S DMT achieves the lowest one. The more the number of split bands, the higher the PAPR. For a CCDF level of $10^{-3}$, the PAPR of the different modulation schemes are listed in Table I.

The noise power spectrum density (PSD) can be measured in DMT transmission. Transmission system with lower PAPR can obtain more output signal power with fixed optical output power. The modulation power gain $G$ is defined as the gap between the system PAPR and the PAPR in DMT. The modulation power gain is considered in the calculation of channel capacities for different modulation schemes.

\section{THEORETICAL RESULTS}

In this section, a theoretical optimization of MB-DFT-S DMT transmission over POF is given. We firstly optimize MBDFT-S DMT system with fixed subcarrier spacing $\Delta f$. With fixed subcarrier spacing $\Delta f$ of $1 \mathrm{MHz}$, each MCM scheme can reach the maximal capacity with its optimized used bandwidth. Then we change the subcarrier spacing $\Delta f$ in order to calculate the system capacity. Finally, practical MCM system transmissions over $50 \mathrm{~m}$ SI-POF employing bit-loading

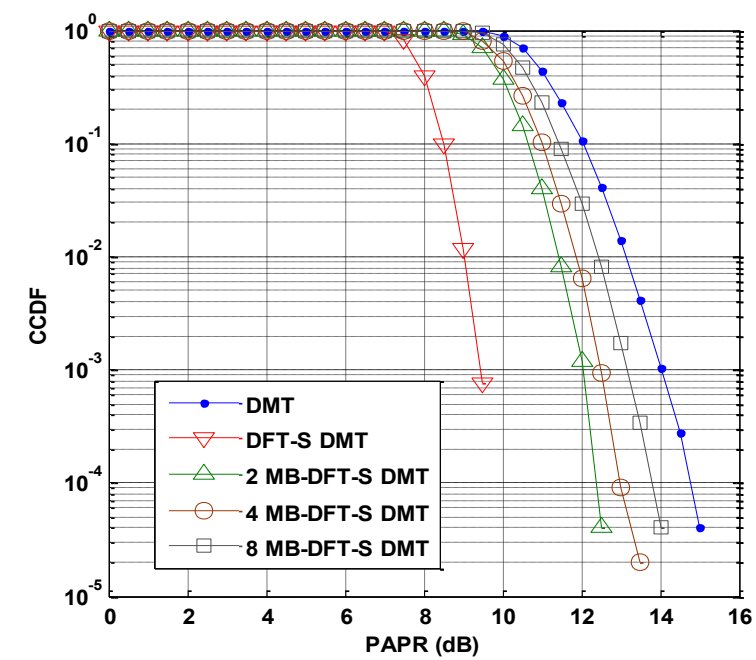

Figure 2. CCDF of DMT, DFT-S DMT and MB-DFT-S DMT schemes with 512 subcarriers

TABLE I. PAPR OF DIFFERENT MODUALTION SCHEMES

\begin{tabular}{|c|c|c|c|c|c|}
\hline $\begin{array}{c}\text { Modulation } \\
\text { Scheme }\end{array}$ & DMT & $\begin{array}{c}\text { DFT-S } \\
\text { DMT }\end{array}$ & $\begin{array}{c}2 \text { Block } \\
\text { Split }\end{array}$ & $\begin{array}{c}4 \text { Block } \\
\text { Split }\end{array}$ & $\begin{array}{c}8 \text { Block } \\
\text { Split }\end{array}$ \\
\hline PAPR & $14 \mathrm{~dB}$ & $9.5 \mathrm{~dB}$ & $12 \mathrm{~dB}$ & $12.5 \mathrm{~dB}$ & $13 \mathrm{~dB}$ \\
\hline
\end{tabular}

technique are presented. All results are obtained with MonteCarlo simulations.

\section{A. MB-DFT-S DMT Optimizations}

\section{1) Used bandwith and number of bands}

As expected, the channel capacity varies with respect to the used bandwidth. Therefore it is important to investigate the theoretical channel capacity with different bandwidths. Given the used bandwidth $W_{\max }$ and subcarrier number $n$, the subcarrier spacing can be expressed as:

$$
\Delta f=W_{\max } / n
$$

In the following investigations, we select $W_{\max }$ from 1 to $512 \mathrm{MHz}$ and fixe $\Delta f$ as $1 \mathrm{MHz}$. The $3 \mathrm{~dB}$ bandwidth $f_{0}$ and noise PSD $N_{0}$ are obtained from measured results of a real 50 $\mathrm{m}$ SI-POF transmission. Specifically, $f_{0}$ is $84 \mathrm{MHz}$ and $N_{0}$ is $112 \mathrm{~dB} / \mathrm{Hz}$ [15]. We assume that total modulated signal power $E_{\text {total }}$ of each MCM scheme is constant. Thus the transmission power of each subcarrier $E_{i}$ is therefore $E_{i}=E_{\text {total }} / n$, where $n$ can vary from 1 to 512 .

Capacities of DMT, DFT-S DMT and MB-DFT-S DMT schemes are investigated employing aforementioned Gaussian channel model provided in (3). Figure 3 presents the POF channel capacity with respect to different used bandwidth $W_{\max }$. It can be seen that each modulation scheme has an optimum bandwidth usage which leads to the maximum capacity. When the used bandwidth ranges from $128 \mathrm{MHz}$ to $160 \mathrm{MHz}$, DFT-S DMT scheme offers the best performance. It reaches to its maximal capacity with the used bandwidth of 160 $\mathrm{MHz}$. However, it is worth noting that the capacity of DFT-S DMT scheme is sensitive to the used bandwidth compared to other schemes. This is shown by the severe capacity 


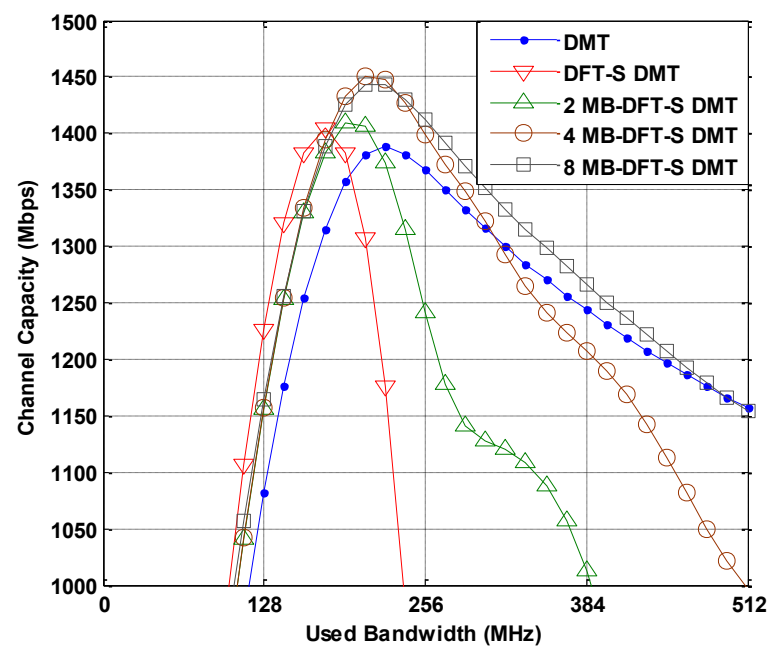

Figure 3. Capacities of DMT, DFT-S DMT and MB-DFT-S DMT schemes: $f_{0}=84 \mathrm{MHz}, N_{0}=-112 \mathrm{~dB} / \mathrm{Hz}$

degradation when system works beyond optimum bandwidth, as demonstrated in Figure 3.

Furthermore, as the PAPR in DFT-S DMT and MB-DFT-S DMT schemes is lower than original DMT modulation, both of them can obtain more system capacity than the original DMT modulation system. In addition, as shown in Figure 3, MBDFT-S DMT systems with 4 or 8 bands both outperform other MCM schemes, offering a maximal system capacity around $1450 \mathrm{Mbps}$ with used bandwidth of $220 \mathrm{MHz}$. Moreover, compared to DFT-S DMT schemes, MB-DFT-S DMT schemes are more efficient with various used bandwidth.

\section{2) Subcarriery frequency spacing}

With the help of optimized used bandwidth, channel capacities of each modulation scheme with different subcarrier frequency spacing are investigated. As subcarrier spacing $\Delta f$ changes from $0.25 \mathrm{MHz}$ to $16 \mathrm{MHz}$, the channel capacities of each modulation scheme with its optimal bandwidth are presented in Figure 4. As shown in the figure, the smaller the subcarrier spacing $\Delta f$, the higher the channel capacity. Furthermore, the capacity lost due to the guide interval in MCM system can also be reduced with smaller subcarrier spacing. However, when $\Delta f$ is less than $0.5 \mathrm{MHz}$, the improvement of channel capacity is less significant. In addition, as subcarrier number $n$ is increasing when $\Delta f$ decreases, larger size of FFT is used, which leads to an increased system complexity. Therefore a $0.5 \mathrm{MHz} \Delta f$ offers a good tradeoff between performance and complexity.

\section{B. MB-DFT-S DMT Attainable Bit-rate with Bit-loading}

MB-DFT-S DMT transmission employing Chow's bitloading algorithm in the aforementioned Gaussian POF channel is simulated. We investigate the relationship between the practical transmission bit-rate and the used bandwidth. The simulation is carried out with a target SER of $1 \cdot 10^{-3}$ and a subcarrier spacing of $0.5 \mathrm{MHz}$.

The practical transmission bit-rate of $50 \mathrm{~m}$ SI-POF employing different DMT modulation schemes is shown in Figure 5. From the simulation results, we can see that, with bitloading technique, both DFT-S DMT and MB DFT-S DMT

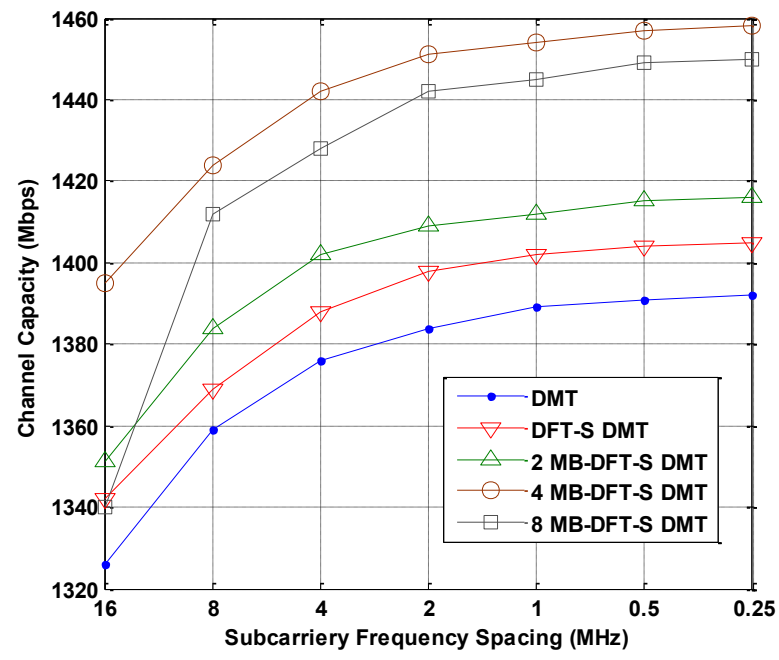

Figure 4. Capacities of optimized MCM schemes with different subcarrier spacing: $f_{0}=84 \mathrm{MHz}, N_{0}=-112 \mathrm{~dB} / \mathrm{Hz}$

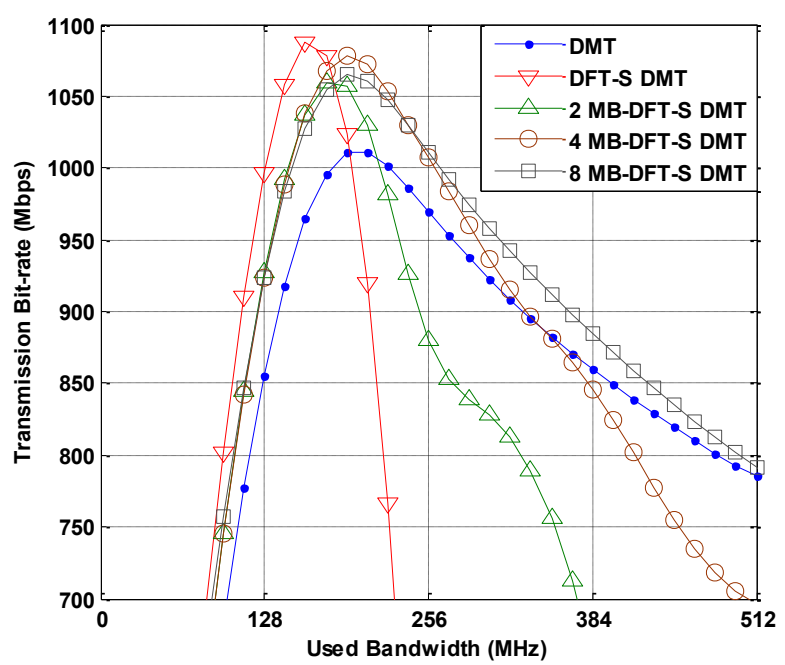

Figure 5. Transmission bit-rate of DMT, DFT-S DMT and MB-DFT-S DMT schemes: $f_{0}=84 \mathrm{MHz}, N_{0}=-112 \mathrm{~dB} / \mathrm{Hz}, \Delta \mathrm{f}=0.5 \mathrm{MHz}$

schemes obtain higher transmission bit-rate than the original DMT scheme. DFT-S DMT scheme achieves similar maximal transmission bit-rate as MB DFT-S DMT schemes. However, the bit-rate of DFT-S DMT scheme is very sensitive to the used bandwidth, which indicates that it is difficult to obtain the optimal performance in the practical use. Furthermore, 4-band and 8-band MB-DFT-S DMT schemes can reach the bit-rate around $1070 \mathrm{Mbps}$, providing 6\% enhancement compared to original DMT scheme.

\section{EXPERIMENTAL RESULTS}

In the previous section, the theoretical performance of DMT, DFT-S DMT and MB-DFT-S DMT schemes in terms of system capacity and transmission bit-rate have been analyzed and simulated. In this section, we present an experimental result in order to compare with the theoretical results. 


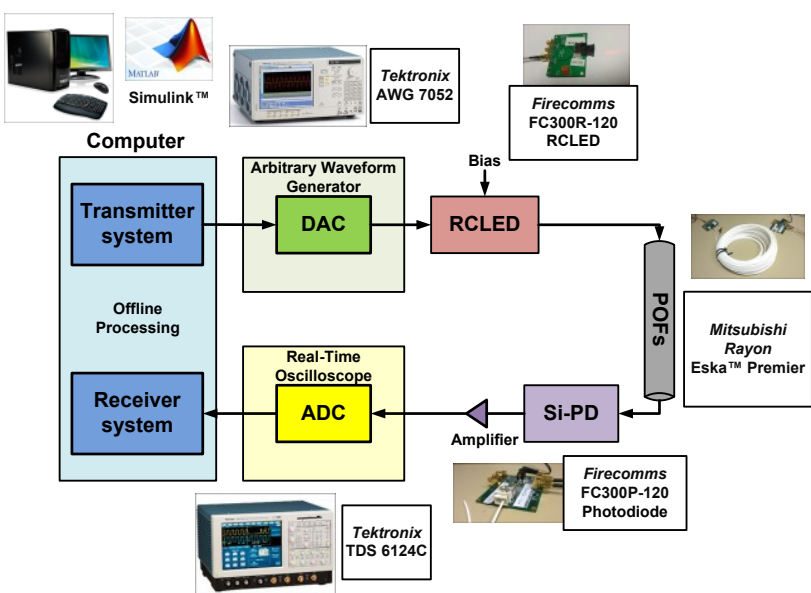

Figure 6. Experimental setup of $50 \mathrm{~m}$ SI-POF transmission system Computer offline processing

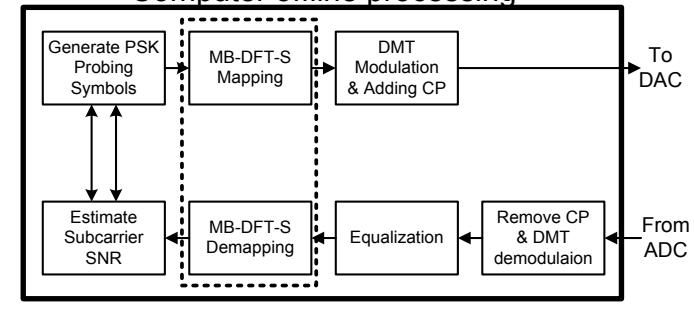

Figure 7. Offline processing of subcarrier SNR estimation

\section{A. Experimental Setup}

The block diagram of the experimental setup of a MBDFT-S DMT over a $50 \mathrm{~m}$ SI-POF channel is depicted in Figure 6 . The signals are generated offline by Simulink ${ }^{\mathrm{TM}} .512$ subcarriers of DMT, DFT-S DMT and MB-DFT-S DMT modulation systems are respectively generated with a 2048 points IFFT block. The first subcarrier is null in order to suppress DC value in the POF transmission system. A cyclic prefix $(\mathrm{CP})$ with 32 symbols is added at the beginning of each IFFT modulated block, which is proved to be sufficient in order to avoid inter-symbol interference [16].

Modulated signals are stored in an arbitrary waveform generator (AWG) and generated by DAC in the AWG. In order to investigate system performance with different used bandwidths, sampling rate of the DAC in AWG is set to 1.25 Giga-samples/s, 1 Giga-samples/s, 0.75 Giga-samples/s and 0.5 Giga-samples/s. Since 512 subcarriers are used in 2048 points IFFT, all generated DMT symbols are transmitted within a used bandwidth of $312.5 \mathrm{MHz}, 250 \mathrm{MHz}, 187.5 \mathrm{MHz}$ and $125 \mathrm{MHz}$, respectively.

After DAC, electrical signal is applied to a resonant cavity light emitting diode (RCLED), which is manufactured by Firecomms ${ }^{\mathrm{TM}}$. A $20 \mathrm{~mA}$ biasing current is set to drive RCLED and $-1 \mathrm{dBm}$ optical output power is obtained. The emitted light is subsequently transmitted via $50 \mathrm{~m}$ SI-POF, $-12 \mathrm{dBm}$ optical power is received at the output of the fiber. Received optical signal is detected by a photodiode and amplified by a transimpedance amplifier (TIA), which is also manufactured by Firecomms ${ }^{\mathrm{TM}}$. Finally, electrical signal is captured by digital oscilloscope (DSO) and stored for offline processing. The receiver DSO is synchronized to the off-line transmitter AWG.

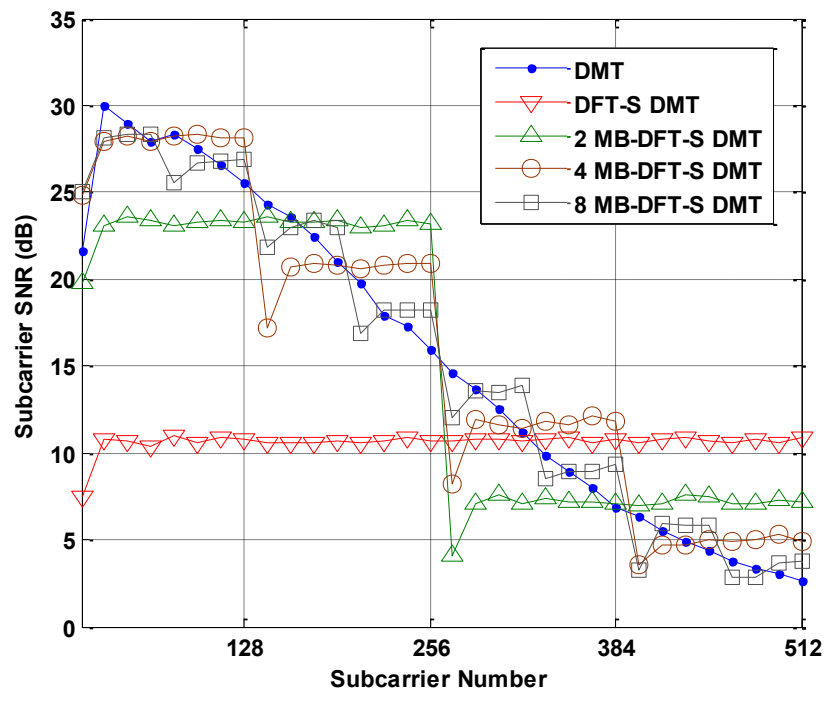

Figure 8. Measured subcarrier SNR in DMT, DFT-S DMT and MBDFT-S DMT systems. Used bandwidth: $250 \mathrm{MHz}$

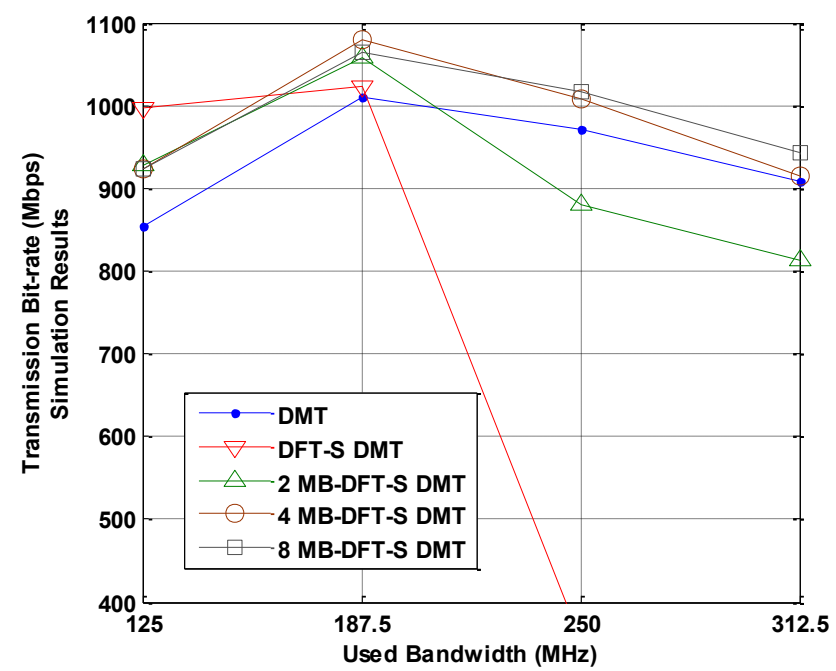

(a)

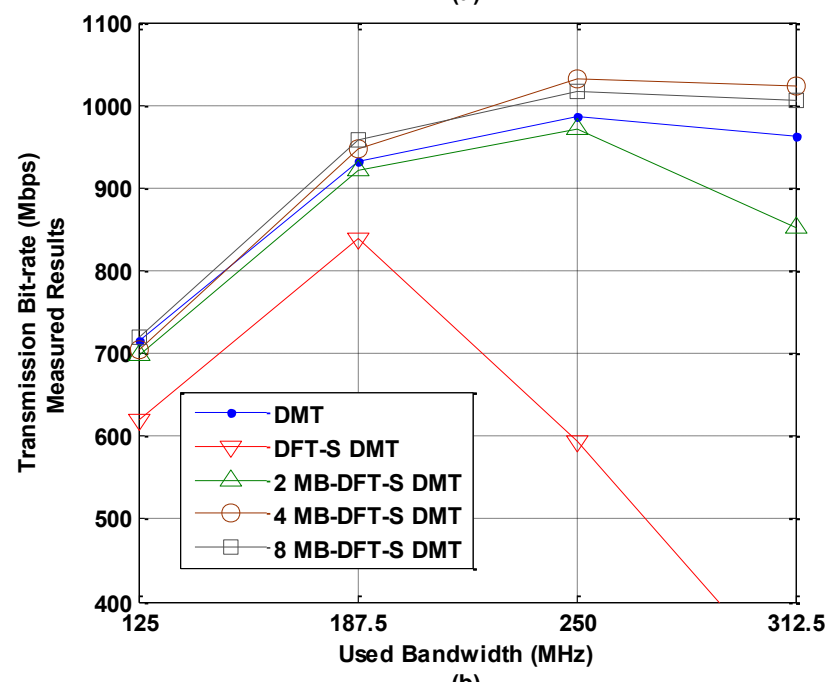

(b)

Figure 9. Simulation and measured results of transmission bit-rate over $50 \mathrm{~m}$ SI-POF with different DMT schemes. (a) Simulation results, (b) Measured results 
The system attained bit-rate is evaluated by the estimated SNR of each subcarrier of received signal. As shown in Figure 7, the offline processing of transmitter and receiver is processed as follows: PSK probing symbols are generated in the transmitter. After MB-DFT-S mapping and DMT modulation, $\mathrm{CP}$ is added. Then, the generated symbols are stored in AWG and converted to analog signal by the DAC in AWG. After POF transmission, received signals from ADC are initially demodulated by FFT. An equalizer is used for channel equalization. After MB-DFT-S de-mapping, subcarrier SNR can be estimated by received symbols according to the known symbol information in transmitter. With the information of subcarrier SNR, available transmission bit is allocated at each subcarrier.

\section{B. Experimental Results and Analysis}

With the aforementioned experimental setup, subcarrier SNR of each modulation scheme is measured after a $50 \mathrm{~m}$ SIPOF transmission. We provide subcarrier SNR measurements with a used bandwidth of $250 \mathrm{MHz}$ in $50 \mathrm{~m}$ SI-POF transmission. As shown in Figure 8, subcarrier SNR of DMT, DFT-S DMT and MB-DFT-S DMT is measured after the equalization. In DFT-S DMT scheme, each subcarrier obtains the same SNR after equalization process, which is shown as a straight line around $11 \mathrm{~dB}$ in Figure 8 . On the other hand, as far as MB-DFT-S DMT scheme is concerned, subcarrier SNR in each band is the same after the equalization. As the whole bandwidth is divided into several parts with different SNR, it is suitable to apply the bit-loading technique in order to achieve overall system capacity.

The comparison of transmission bit-rates between theoretical and experimental results is depicted in Figure 9. Four used bandwidth of $312.5 \mathrm{MHz}, 250 \mathrm{MHz}, 187.5 \mathrm{MHz}$ and $125 \mathrm{MHz}$ are evaluated in POF transmissions. As shown in the figure, 4-bands and 8-bands MB-DFT-S DMT schemes obtain the best performance in both simulation and experimental results. They provide the highest transmission bit-rate around $1030 \mathrm{Mbps}$. The improvement is near $6 \%$ compared to original DMT scheme which has maximal transmission bit-rates around 960 Mbps. In general, the experimental results agree with theoretical analysis, except that the optimal used bandwidth is measured around $250 \mathrm{MHz}$ in a real POF channel. The differences are mainly due to the deviation of the Gaussian channel model from the practical POF channel. Furthermore, in a real POF transmission, DFT-S DMT scheme performs worse than theoretical results when used bandwidth is $187.5 \mathrm{MHz}$. DFT-S DMT can only obtain 820 Mbps bit-rate, compared to the theoretically estimated value of $1020 \mathrm{Mbps}$. The difference is mainly due to the non-linearity behavior in POF transmission system. Therefore, we can conclude that DFT-S DMT scheme significantly suffers from non-linearity distortion. However, MB-DFT-S DMT schemes show stronger robustness against non-linearity, which means that MB-DFT-S DMT is a more promising solution in low-cost POF transmission systems.

\section{CONCLUSION}

In this paper, the MB-DFT-S DMT scheme is proposed and optimized for POF communication systems taking into account "dimensioning" of the global system as well as bit-loading. Compared to the classical DMT scheme, MB-DFT-S DMT scheme owns lower PAPR, which can inherently enhance modulated signal power with the same optical output power. Theoretical analysis shows that MB-DFT-S DMT schemes can obtain higher channel capacities than DMT systems. Furthermore, with more subcarriers more channel capacity can be obtained. Employing bit-loading technique, both simulation and experimental results show that 4-bands and 8-bands MBDFT-S DMT schemes outperform other MCM schemes in $50 \mathrm{~m}$ SI-POF transmissions with the same multi-carrier system setup and output optical power. The measured results prove the advantage of MB-DFT-S DMT scheme in the typical Gaussian-shaped POF channel. In addition, compared to DFTS DMT, the proposed MB-DFT-S DMT scheme is shown to be more efficient in presence of non-linearity mitigation, which means it is a promising technique for the practical POF transmission systems.

\section{REFERENCES}

[1] C.M. Okonkwo, et al., "Recent Results from the EU POF-PLUS Project: Multi-Gigabit Transmission over $1 \mathrm{~mm}$ Core Diameter Plastic Optical Fibers", J. Lightw. Technol., Vol. 29, No. 2, pp. 186-193, Jan. 15, 2011.

[2] M. Popov, "Recent Progress in Optical Access and Home Networks: Results from the ALPHA Project”, in Proc. ECOC 2011.

[3] A.M.J. Koonen, et al., "Cost Optimization of Optical In-Building Networks", in Proc. ECOC 2011.

[4] O. Ziemann, et al., "POF Handbook: Optical Short Range Transmission Systems", 2nd Edition, Springer, 2008.

[5] S. Randel, et al., "Advanced Modulation Schemes for Short-Range Optical Communications", IEEE J. Select. Topics in Quantum Electronics, Vol. 16, No. 5, Sep./Oct. 2010.

[6] D. Visani, et al., "Beyond 1 Gbit/s Transmission Over $1 \mathrm{~mm}$ Diameter Plastic Optical Fiber Employing DMT for In-Home Communication Systems", J. Lightw. Technol., Vol.29, No.4, pp.662-628, Feb.15, 2011.

[7] H. Yang, et al., " $47.4 \mathrm{~Gb} / \mathrm{s}$ Transmission Over $100 \mathrm{~m}$ Graded-Index Plastic Optical Fiber Based on Rate-Adaptive Discrete Multitone Modulation", J. Lightw. Technol., Vol. 28, No. 4, Feb.15, 2010.

[8] S. Karabetsos, et al., "DFT-Spread DMT Modulation for 1-Gb/s Transmission Rate Over $100 \mathrm{~m}$ of 1-mm SI-POF", IEEE Photon. Technol. Lett., Vol. 24, No. 10, pp. 836 - 838, May 15, 2012.

[9] H.G. Myung, J. Lim, and D.J. Goodman, "Single Carrier FDMA for Uplink Wireless Transmission”, IEEE Vehicular Technol. Mag., Vol. 1, No. 3, pp. 30-38, Sep. 2006

[10] Y. Tang, W. Shieh, and B.S. Krongold, "Capacity of Single Carrier System with Frequency-Domain Equalization", in Proc. IEEE 6th CAS Symp. On Emerging Technologies: Mobile and Wireless Comm., pp. 429-432, May 2004

[11] Y. Tang, W. Shieh, and B.S. Krongold, "DFT-Spread OFDM for Fiber Nonlinearity Mitigation", IEEE Photon. Technol. Lett., Vol. 22, No. 16, pp. 1250 - 1252, Aug.15, 2010.

[12] Q. Yang, Z. He, Z. Yang, S. Yu, X. Yi, A.A.Amin, and W. Shieh, "Coherent Optical DFT-Spread OFDM in Band-multiplexed Transmissions", in Proc. ECOC 2011.

[13] A. Garcia-Armada, "SNR gap approximation for M-PSK-based bitloading”, IEEE Trans. on Wireless Comm., vol. 5, pp. 57-60, 2006.

[14] P. S. Chow, J. M. Cioffi, and J. A. C. Bingham, "A practical discrete multitone transceiver loading algorithm for data transmission over spectrally shaped channels", IEEE Trans. Commun., Vol. 43, No. 2, pp. 773-775, Feb. 1995

[15] B. Cardiff, M.F. Flanagan, F. Smyth, L.P. Barry and A.D. Fagan, "On Bit and Power Loading for OFDM Over SI-POF”, J. Lightw. Technol., Vol. 29, No. 10, pp. 1547-1554, May 15, 2011.

[16] L. Peng, S. Haese, M. Helard, "Optimum configuration for discrete multi-tone transmission over polymer optical fiber", in Proc. ICT 2012. 